\title{
Infertility in the high-producing dairy cow: Where is the light at the end of the tunnel?
}

M. C. Lucy

University of Missouri, Columbia, Missouri, United States

Email:lucym@missouri.edu

Introduction Dairy cattle have been traditionally selected for milk production and milk components (fat and protein). The traditional approach made sense because farmers are paid for milk. Dairy selection has changed in the past decade. Secondary traits, including reproduction traits, are being included in indices by specifically decreasing the emphasis on production. Greater emphasis on non-production traits reflects the industry desire for functional dairy cattle with greater fertility. The hope is that appropriate economic weightings are placed on secondary traits so that slowed progress in milk production is offset by improved farm economics created by more functional cows. Few will argue that high fertility has value. The real question is how much value (economic weight) does it have? This later question is particularly difficult when one considers that selection indices are designed for use by the national dairy herd that is comprised of increasingly diverse production systems.

Postpartum body condition and fertility Regardless of system, modern dairy cows are thinner than their ancestors. Given limited capacity to consume adequate dietary energy, dairy cows mobilize fat to support lactation. Improvements in milk production occur more rapidly than improvements in the capacity for cows to consume additional energy or the willingness of farmers to provide more feed. Thus, body fat is the obvious source of the additional energy. Once dairy cows begin lactation, they will migrate toward their body condition set point through the coordinated control of both feed intake and the depletion of adipose tissue. Their body condition during lactation affects their reproductive performance. Cows with a high body condition during lactation have high fertility whereas cows with a low body condition during lactation have low fertility. Reproductive tissues sense energy demands and function poorly during low body condition.

Hormonal links between body condition and fertility Growth hormone (GH) is an anabolic hormone that is increased during early lactation. It has the capacity to antagonize insulin action. Antagonizing the actions of insulin has a nutrient partitioning effect through which the production of milk is favoured. High producing dairy cows have high concentrations of GH and low concentrations of insulin. Dairy cows also suffer from insulin resistance (insensitivity to insulin manifested at the tissue level). The large increase in circulating GH concentrations during early lactation drives body condition loss. After this initial period of GH action, there is a second period where GH remains elevated. The long-term steady-state blood GH concentrations may ultimately determine the body condition for the individual cow because GH antagonizes lipogenesis. Many of the mechanisms that control reproduction are linked directly to the nutritionally-controlled hormonal milieu of the animal. Secretion of LH and FSH is controlled by GnRH from the hypothalamus. Postpartum cows will begin to cycle when energy balance improves and LH pulsatility reaches a critical level. Insulin and IGF1 concentrations gradually increase postpartum as well. Cows in negative energy balance have lower blood concentrations of insulin and IGF-I. Insulin and IGF-I stimulate GnRH secretion from the hypothalamus and LH secretion from the pituitary. The hormonal control of GnRH and LH, therefore, arises from the metabolic and nutritional status of the animal via insulin and IGF1. These peripheral metabolic hormones will act on the hypothalamus to convey information from metabolically important tissues. A variety of metabolites (glucose, nonesterified fatty acids, etc.) and other hormones may also be involved. The same metabolites and hormones that influence GnRH secretion and ultimately LH and FSH secretion may act directly on the ovary to influence the sensitivity of the ovary to LH and FSH. Thus, the effects of nutrition on reproduction are manifested at the ovary and at the pituitary and hypothalamus through metabolic hormones (GH, IGF1, and insulin) that are essential for nutrient partitioning. In addition to the follicle, the corpus luteum, uterus, and embryo respond positively to insulin and IGF1. Nutrient partitioning for greatest milk production occurs when blood GH is elevated and blood insulin and IGF1 are low. Improvements in reproduction occur under a contrasting hormonal milieu (i.e., high blood insulin and IGF1 concentrations). It is difficult, therefore, to consistently achieve good reproduction in cows that undergo extremes in nutrient partitioning and have low insulin and IGF1.

Rethinking the lactation curve An interesting question that could be raised is whether or not the dairy industry should attempt to change the lactation curve of the cow to relieve some of the body condition loss in early lactation. If peak milk production was less and persistency was greater then the overall level of production across the entire lactation may not change (loss of milk at peak being compensated by greater production in later lactation). Manipulating the lactation curve in this manner for the purpose of alleviating body condition loss is not a new concept and was proposed in 1985. Peak milk production, ascent to peak production, and persistency are traits with moderate heritability so the shape of the lactation curve can theoretically be changed. Recent publications have raised the possibility of increasing persistency and decreasing peak milk yield as a means to alleviate losses in body condition.

Conclusions Reproductive traits are being included in selection indices worldwide by decreasing the emphasis on production. Greater emphasis on reproductive traits reflects the industry desire for more functional and efficient dairy cattle. The traditional view of an "efficient cow" was one that mobilized body fat in early lactation so that peak milk production was maximized. This approach to efficiency has apparently antagonized reproduction through changes in metabolic hormones (GH, IGF1, and insulin) and metabolites (glucose and nonesterified fatty acids). Changing the shape of the lactation curve so that peak milk production is less and persistency of lactation is greater may improve reproductive function while maintaining total lactation yield. 